Histoire de l'éducation

DEEDCATION

$147 \mid 2017$

Les lieuX de l'enseignement technique $\left(\mathrm{XIX}^{\mathrm{e}}-\mathrm{XX}^{\mathrm{e}}\right.$ siècles)

\title{
Les écoles professionnelles en Suisse : palais ou
} usines?

Vocational schools in Switzerland: palaces or factories?

Dave Lüthi

\section{OpenEdition}

\section{Journals}

Édition électronique

URL : https://journals.openedition.org/histoire-education/3300

DOI : $10.4000 /$ histoire-education.3300

ISSN : 2102-5452

Éditeur

ENS Éditions

\section{Édition imprimée}

Date de publication : 30 juin 2017

Pagination : 119-146

ISSN : 0221-6280

\section{Référence électronique}

Dave Lüthi, «Les écoles professionnelles en Suisse : palais ou usines? », Histoire de l'éducation [En ligne], 147 | 2017, mis en ligne le 30 juin 2020, consulté le 20 mai 2021. URL : http://

journals.openedition.org/histoire-education/3300 ; DOI : https://doi.org/10.4000/histoire-education. 3300 


\title{
Les écoles professionnelles en Suisse : palais ou usines?
}

\author{
Dave Lüthi
}

La question de l'architecture des écoles professionnelles demeure en Suisse une terra incognita. L'histoire complexe du développement de l'enseignement professionnel, fractionné entre les différentes entités politiques de la confédération, explique en grande partie le désintérêt dont cette architecture fait l'objet à ce jour ${ }^{1}$, alors même que l'architecture scolaire connaît depuis plusieurs décennies les faveurs des chercheuses et des chercheurs ${ }^{2}$. La multiplicité des types d'écoles professionnelles joue également un rôle dans cette méconnaissance, leur variété même pouvant apparaître comme un frein à l'inventaire ${ }^{3}$, d'une part, mais surtout à leur analyse architecturale, d'autre part. Comment

1 À ce sujet, voir notamment : Emil Wettstein et al., La formation professionnelle en Suisse, Lucerne/ Neuchâtel, Conférence des offices cantonaux de formations professionnelles, 1986; Jean-Pierre Tabin, Formation professionnelle en Suisse. Histoire et actualité, Lausanne, Réalités sociales, 1989; Pierre-Yves Donzé, "Culture technique et enseignement professionnel dans les écoles d'horlogerie suisses (1850-1920)", Histoire de l'éducation, nº119, 2008, p. 5-28. Il est à souligner qu'aucun de ces auteurs ne soulève la question de la construction des écoles professionnelles, encore moins de leur architecture.

2 Par exemple pour le canton de Vaud : Geneviève Heller, Marianne Fornet, La cage dorée : de la chambre d'école au groupe scolaire : deux siècles d'architecture vaudoise, Chapelle-sur-Moudon, Ketty \& Alexandre, 1997; Dave Lüthi (dir.), Lausanne-les écoles, Berne, Société d'histoire de l'art en Suisse, 2012.

3 Le corpus entier reste à formuler : afin de dresser une liste exhaustive des établissements au moins jusqu'aux années 1960, l'énumération des différents systèmes scolaires cantonaux et des écoles en dépendant publiée dans Archiv für das schweizerische Unterrichtswesen, vol. 51-52, 1965-1966, p. 23-288, semble une base fiable. 
comparer, en effet, une école ménagère avec une école d'horlogerie, une école d'ingénieurs avec une école d'agriculture? Alors que le palais de la Renaissance sert de modèle à certaines, c'est l'usine qui est la référence pour d'autres, ces deux extrêmes enserrant une hiérarchie subtile et complexe, qu'il faut lire de manière à la fois typologique mais aussi chronologique. À partir d'un corpus encore bien incomplet, mais sans doute représentatif ${ }^{4}$, cette étude s'intéresse aux tendances de fond, sans pouvoir se pencher sur l'histoire institutionnelle ${ }^{5}$ et architecturale de chaque édifice, le travail étant colossal ${ }^{6}$.

\section{Des débuts difficiles}

L'histoire de l'apprentissage professionnel en Suisse est liée à deux facteurs essentiels : d'une part, la géographie des industries, déterminantes dans l'apparition et le développement des écoles, d'autre part, la faiblesse du jeune état fédéral de 1848 quant aux questions de formation - celle-ci restant encore de nos jours du ressort des cantons, sauf pour les deux écoles polytechniques de Zurich et de Lausanne. De fait, dans les débats parlementaires, la question du manque de formation des jeunes travailleurs revient fréquemment, avancée par des députés qui sont aussi souvent chefs d'entreprise. Les intérêts sociaux, défendus par le gouvernement par le biais de la loi fédérale sur les fabriques du 23 mars 1877 qui interdit le travail des enfants de moins de 14 ans et limite la journée de travail à 11 heures, ne sont pas sans servir les intérêts du patronat. En effet, l'Assemblée fédérale demande en 1882 l'ouverture d'une enquête sur l'état des industries en vue, notamment, de créer des écoles des arts et métiers pour relever le niveau de l'industrie helvétique, alors que l'on discute d'une loi sur l'apprentissage professionnel. Cette requête ne tient pas du hasard. La Grande Dépression et la concurrence internationale mettent à

4 Notre inventaire se fonde sur un corpus de 66 bâtiments, à partir du dépouillement des publications suivantes : Inventaire suisse d'architecture 1850-1920 [désormais INSA], 10 vol., Berne, Société d'histoire de l'art en Suisse, 1982-2004; Guide d'architecture suisse 1920-1990 [désormais GAS], 3 vol., Berne, Werk Verlag, 1992-1996; Neues Bauen in der Schweiz : Führer zur Architektur der 20er und $30^{e r}$ Jahre, 2 vol., Blauen, Schweizer Baudokumentation, 1985-1993. Une recherche a également été effectuée dans les principales revues d'architecture suisses sur le portail e-periodica.ch : Bulletin technique de la Suisse romande; Schweizerische Bauzeitung [désormais SBZ]; Das Werk; Werk, Bauen und Wohnen, etc.

5 De nombreuses études, souvent jubilaires, documentent l'histoire individuelle des écoles.

6 À titre d'exemple, nous renvoyons à notre étude : "L'école-usine. L'École des métiers de Lausanne : chronique d'une (très) lente gestation ", Monuments vaudois, nº 4, 2013, p. 57-65. 
mal plusieurs industries majeures du pays ${ }^{7}$; un réveil industriel doublé d'une professionnalisation des métiers techniques est appelé de leurs vœux par nombre de parlementaires. Toutefois, le système politique suisse fédéraliste ne favorise pas la création d'écoles centralisées. Hormis l'École polytechnique de Zurich (1855), aucune autre école fédérale n'est ouverte - l'Université souhaitée par les parlementaires en 1848 restera lettre morte, la tâche étant rapidement déléguée aux cantons qui développent ou transforment les anciennes académies durant le dernier quart du siècle ${ }^{8}$; en dépit des frais engendrés par la croissance rapide de certaines d'entre elles, la création des universités apparait en fait comme un véritable privilège pour les cantons abritant les institutions, qui avaient lutté contre le projet fédéral d'une académie unique. L'enquête amènera, en 1884, à un arrêté fédéral décrétant le subventionnement des classes professionnelles masculines (les féminines le seront en 1895).

Ce paysage politique et institutionnel engendrera la création de nombreuses écoles professionnelles de niveau inférieur et moyen, ne délivrant pas toujours des diplômes mais des brevets fédéraux - très réputés au demeurant -, écoles dont le soutien public est très variable d'un cas à l'autre. De l'école des métiers cantonale à l'école professionnelle fondée par une entreprise ou un groupe d'entreprises, l'éventail des possibilités est varié. Si l'institutionnalisation des établissements se généralise au cours du XX $\mathrm{X}^{\mathrm{e}}$ siècle, elle n'est pas déterminante dans la construction d'édifices $a d$ hoc, les écoles liées à des usines étant parfois mieux dotées que les établissements publics. En revanche, le soutien financier public voire le passage de ces écoles en mains communales est souvent essentiel pour assurer leur survie en période de crise. Pour schématiser les choses, on pourrait dire que les écoles les plus proches des domaines industriels les plus florissants (horlogerie, machines) sont souvent de fondation privée alors que celles qui sont généralistes ou liées à des activités moins rentables émanent respectivement des autorités communales (écoles des métiers, écoles

7 Pierre-Yves Donzé, "Culture technique....", art. cit., p. 11-12.

8 Zurich (1833) et Berne (1834) exceptées, la plupart des universités suisses sont fondées sous leur forme moderne dans le dernier quart du siècle : Genève (1873), Fribourg (1889, première université catholique de Suisse), Lausanne (1890), Neuchâtel (1909). Seconde vague un siècle plus tard : SaintGall (1995), Suisse italienne (1996), Lucerne (2000), toutes trois en terres catholiques. $C f$. Sebastian Brändli, "Université", in Dictionnaire historique de la Suisse [désormais DHS], version en ligne : <http://www.hls-dhs-dss.ch/textes/f/F10418.php> (consulté le 12 octobre 2016). 
professionnelles commerciales ${ }^{9}$ ) ou cantonales (technicums, écoles d'agriculture, écoles ménagères), cette répartition des tâches pouvant évoluer dans le temps ${ }^{10}$.

\section{Les premières écoles professionnelles : les écoles d'horlogerie}

Le cas des écoles d'horlogerie est particulièrement représentatif de la première phase des écoles professionnelles en Suisse ${ }^{11}$. Fondées pour la plupart par des syndicats patronaux - à l'exception notable de la plus précoce, celle de Genève -, elles sont prévues pour l'apprentissage des différentes branches de l'établissage ${ }^{12}$ par une population généralement pauvre; l'école prend souvent un tour philanthropique, auquel l'architecture répond de deux manières différentes. À Genève (1874-1878) ${ }^{13}$, l'école financée par le don du duc de Brunswick et réalisée par les autorités communales après concours prend des allures palatiales : le plan en $\mathrm{E}$ et l'architecture néo-Renaissance (notamment l'aile de la bibliothèque avec fenêtres en plein-cintre et pilastres rythmant les façades) renvoient aux exemples contemporains de collèges, dont l'architecte, Jean Franel, est un bon connaisseur. Le plan en peigne "s'imposait essentiellement pour permettre la multiplication des faces ${ }^{14}$ permettant un éclairage optimal des ateliers-salles de classe. Mais il faut ici souligner une caractéristique a priori contraire aux règles alors très prisées de l'hygiénisme, mais étroitement liée aux besoins spécifiques de l'horlogerie : les classes-ateliers sont alignées contre les façades nord et est des ateliers, alors que les couloirs assez étroits qui les distribuent sont éclairés au sud est à l'ouest. Les ateliers s'affirment clairement en façade par des bandeaux de larges fenêtres séparées par des meneaux de pierre dont

9 Qu'il ne faut pas confondre avec les écoles de commerce; en allemand, on les nomme respectivement Kaufmännische Berufsschule et Handelschule.

10 Suite à la loi fédérale de 1995, entre 1996 et 2003, les écoles professionnelles se sont muées en Hautes écoles spécialisées, entrainant ainsi une répartition nouvelles des tâches. Cf. Karl Weber et al., Différentes, mais de même valeur? De l'évolution des hautes écoles spécialisées suisses, Bâle/ Berne, Gebert Rüf Stiftung, 2010.

11 Pierre-Yves Donzé, "Culture technique...", art. cit.

12 Établissage : division du travail en vigueur dans l'arc jurassien consistant en la fabrication des pièces par des ateliers spécialisés, réunies ensuite pour la finalisation de l'objet par un autre horloger. Sur ce point, voir Pierre-Yves Donzé, Les patrons horlogers de La Chaux-de-Fonds dynamique sociale d'une élite industrielle, 1840-1920, Neuchâtel, Alphil, 2007.

13 INSA, vol.4, p. 365; Les monuments d'art et d'histoire du canton de Genève, vol.2, Berne, Société d'histoire de l'art en Suisse, 2011, p. 267-270.

14 Les monuments d'art et d'histoire du canton de Genève, p. 268. 
l'origine n'est pas scolaire, mais industrielle : le modèle, tant en plan qu'en élévation, c'est bien l'atelier d'horlogerie ${ }^{15}$ (Ill. 1). Les écoles de l'arc jurassien conçues par des regroupements patronaux ne parlent pas un autre langage : à Bienne $(1876)^{16}$ (Ill. 2) et La Chaux-de-Fonds (Hans Mathys, 1885) ${ }^{17}$, les deux écoles s'assimilent presque à des petites usines, n'était leur symétrie de composition, affirmée par un avant-corps à trois axes couronné respectivement d'une lucarne à fronton et d'une horloge, cette dernière formant un lien évident entre l'architecture de l'usine et celle de l'école : l'école-usine, en fait. C'est toutefois à une école unique en son genre, celle des apprentis en métallurgie de Winterthour (Albert Pfister, 1892), que revient la palme de l'école la plus industrielle du corpus helvétique, celle où la fonction scolaire s'efface devant les nécessités très spécifiques de cette branche industrielle ${ }^{18}$ (Ill. 3). On notera que les écoles d'infirmières contemporaines ressemblent également plus à de petits hôpitaux qu'à des écoles ${ }^{19}$.

Comme de manière générale dans le domaine scolaire en Suisse après 1900, l'architecture des écoles d'horlogerie devient moins expressive quant à leur fonction. La vogue du régionalisme (Heimatstil), lancée par les grands événements patriotiques contemporains (notamment l'exposition nationale de Genève en 1896), renouvelle complètement les modes expressifs des édifices servant à l'édification morale des citoyens - écoles, églises en particulier - et ce pour un demi-siècle. Comme pour les autres types d'écoles étudiés plus loin, la référence au monde industriel disparaît peu à peu au profit de modèles soi-disant traditionnels, qui véhiculent des valeurs conservatrices qui ne sont pas anodines. En effet, une partie de la bourgeoisie commerçante regrette le corporatisme en vigueur sous l'Ancien Régime ${ }^{20}$ ainsi que le Verlagssystem ${ }^{21}$ et cherche à les

15 Il faut préciser ici que l'architecture industrielle horlogère n'en est alors qu'à ses balbutiements.

16 INSA, vol. 3, p. 100.

17 Ibid., p. 201. L'aile septentrionale ajoutée en 1901 accusera le caractère industriel de l'école.

18 INSA, vol. 10, p. 174.

19 Comme à Zurich, la Pflegerinnenschule, 1901 (INSA, vol. 10, p. 321).

20 Lors de l'élaboration de la loi fédérale de 1930 sur la formation professionnelle, on déplore toujours la perte de ce "bon vieux temps" mythifié : "Lorsque les corporations de métiers eurent disparu en Suisse, la formation professionnelle y resta en maints endroits négligée [...]". Cf. Feuille fédérale, 14 novembre 1928, nº46, p.763, cité par Jean-Pierre Tabin, Formation professionnelle..., op. cit., p. 81 .

21 Production décentralisée basée sur le travail à domicile, caractéristique de l'industrie suisse protoindustrielle. Cf. Ulrich Pfister, "Verlagssystem", in DHS : <http://www.hls-dhs-dss.ch/textes/f/ F13880.php>(consulté le 12 octobre 2016). 

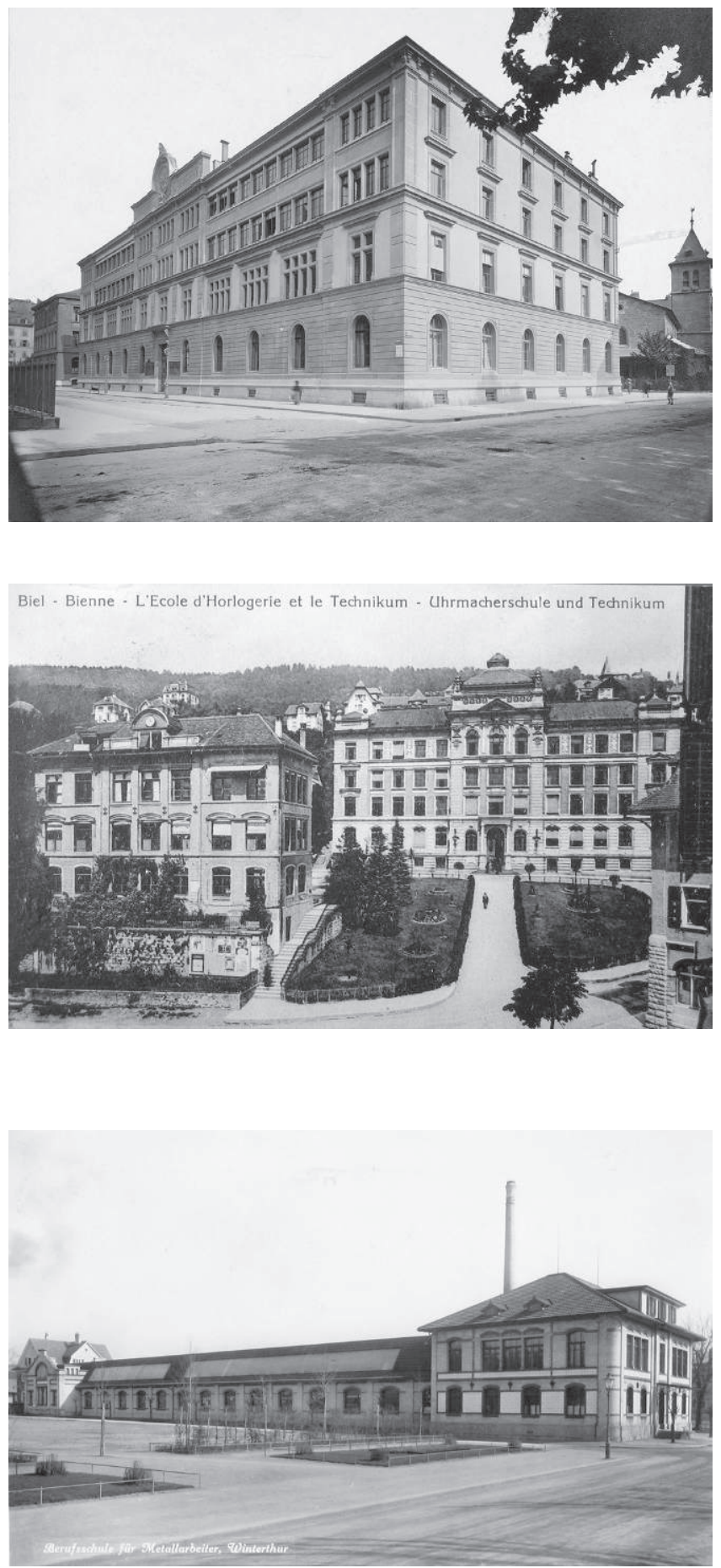

Ill. 1 : Genève, école d'horlogerie. Photographie anonyme, ap. 1878

Source : Centre d'iconographie de la Bibliothèque de Genève, IG 2000-003-44.

Ill. 2 : Bienne, école d'horlogerie et technicum. Carte postale, s.d. (ca. 1919)

Source : Zurich, ETH-Bibliothek, Bildarchiv, Fel_004715-RE.
Ill. 3 : Winterthour, école de métallurgie. Carte postale, s.d. (ca. 1929)

Source : Zurich, ETH-Bibliothek, Bildarchiv, Fel_008195-RE. 
remettre en vigueur, au moins par des références architecturales plus ou moins explicites. À l'instar de l'école d'horlogerie de la Vallée de Joux (Arthur Lerch, 1908) ${ }^{22}$, les formes de ces établissements se trouvent caractérisées durant plusieurs décennies par cette idéologie très forte : à quelques exceptions près, l'architecture ne s'en défera qu'après la Seconde Guerre mondiale.

\section{Les technicums}

Dans les villes industrielles, des écoles techniques appelées technicums, sur le modèle germanique, voient le jour dès les années 1870 mais surtout entre 1893 et 1902. Celui de Winterthour, ouvert en 1876, est à tous points de vue fondateur en la matière : il servira de modèle institutionnel à tous les autres et demeurera jusqu'à l'époque contemporaine un moteur incontesté en matière d'enseignement de l'ingénierie ${ }^{23}$. Il se situe dans la seconde ville du canton de Zurich, qui tire sa richesse de l'industrie textile et, de plus en plus à la fin du $\mathrm{XIX}^{\mathrm{e}}$ siècle, de la construction de machines dans des entreprises connues mondialement (Rieter, Sulzer et Schweizerische Lokomotiv- und Maschinenfabrik). La ville connaît alors un essor important, sa population passant de moins de 20000 habitants en 1870 à près de 41000 en 1900. L'établissement de Winterthour est le descendant d'une première école technique fondée en 1866 par l'ingénieur en machines Friedrich Autenheimer. Ce dernier ambitionnait la création d'un Technicum suisse (Technikum für die Schweiz) dont les compétences se situeraient à mi-chemin entre une école des métiers et l'École polytechnique fédérale récemment ouverte à Zurich ${ }^{24}$. Autenheimer cherche à former des ingénieurs de niveau médian, sans faire concurrence à l'école fédérale, mais en se plaçant clairement dans son sillage et dans celui de son principal modèle, la Königlich Württembergische Baugewerkschule (École royale de construction du Wurttemberg) de Stuttgart, fondée en 1845. Le technicum devait être, quant à lui, créé par les autorités communales, proches des milieux patronaux, mais c'était sans compter avec un refus populaire lors de la votation cantonale de 1872. Le projet accepté lors d'un nouveau scrutin en 1874 n'est

22 Feuille d'Avis de Lausanne, 26 avril 1907; Chantal Schindler, 75e anniversaire de l'Ecole technique la Vallée de Joux Le Sentier : 1901-1976, Le Sentier, École technique de la Vallée de Joux, 1976.

23 La loi fédérale de 1995 sur les hautes écoles spécialisées résulte en partie des revendications de cette institution.

24 INSA, vol. 10, p.43-44. 
plus communal, mais cantonal; il reçoit par conséquent des subventions qui lui assurent son développement à venir ${ }^{25}$. Sans doute, cette école sert aussi de rééquilibrage entre les deux principales villes du canton : Zurich l'intellectuelle avec son université et son École polytechnique, Winterthour l'industrieuse avec son technicum et son école des métiers (Gewerbeschule) ${ }^{26}$. D’autres fondations de technicums suivront à la fin du siècle à l'ouest du pays, à Bienne (1890), Berthoud (1892), Fribourg (1896), Genève (1901).

Le technicum de Winterthour (1877-1878) offre une architecture qui exprime l'ambition de ses fondateurs (Ill. 4). Le corps de bâtiment principal rectangulaire est flanqué deux courtes ailes formant saillie sur les deux longues façades. Le plan est celui d'une école : un couloir longitudinal distribue les salles de part et d'autre alors que l'axe central est occupé par le vestibule d'entrée et la cage d'escalier. Côté cour, un porche à triple arcade d'ordre dorique marque l'entrée principale, la travée centrale étant en outre dotée d'un petit fronton porté par deux colonnes supplémentaires. À l'arrière, les deux ailes, sensiblement plus larges, entourent le musée industriel conçu comme un corps bas branché sur la partie centrale, terminé par une abside polygonale. À l'intérieur, une galerie permet l'exposition de nombreux objets industriels illuminés par une verrière ménagée dans la toiture. L'architecture des façades fait référence à la Renaissance italienne, selon un modèle alors couramment appliqué en Allemagne et en Suisse dans l'architecture scolaire. Le projet avait été confié à l'architecte communal Joseph Bösch (1839-1922) mais après sa nomination comme professeur au technicum, c'est vers son successeur, Theodor Gohl (18441910), qu'on se tourne pour le projet définitif. Le chantier débute en 1877 par le bâtiment de chimie, situé à proximité du bâtiment principal, terminé quant à lui en janvier 187927. Gohl, comme Bösch d'ailleurs, est un élève de Gottfried Semper dont il répète à l'envi la leçon architecturale - soin du choix des matériaux, texturation des façades, style néo-Renaissance, monumentalité relative du $\operatorname{plan}^{28}$. À peine inauguré, l'édifice est publié dans la revue polytechnique

25 Les principales disciplines enseignées sont : construction, ingénierie en machines, commerce; la chimie, la géométrie et les arts industriels seront abandonnés en 1915. S'y ajoutent peu à peu l'électrotechnique, la formation des employés des chemins de fer, les travaux publics (INSA, vol. 10, p. 43).

26 Sur cette école au destin complexe, voir INSA, vol. 10, p. 43.

27 Louis Calame, Das kantonale Technikum in Winterthur 1874-1924. Zur Feier des füngzigjährigen Bestehens, Winterthour, Binkert, p. 13.

28 Il aura l'occasion de construire de nombreux édifices publics comme architecte à la Direction des constructions fédérales dès 1891 . 

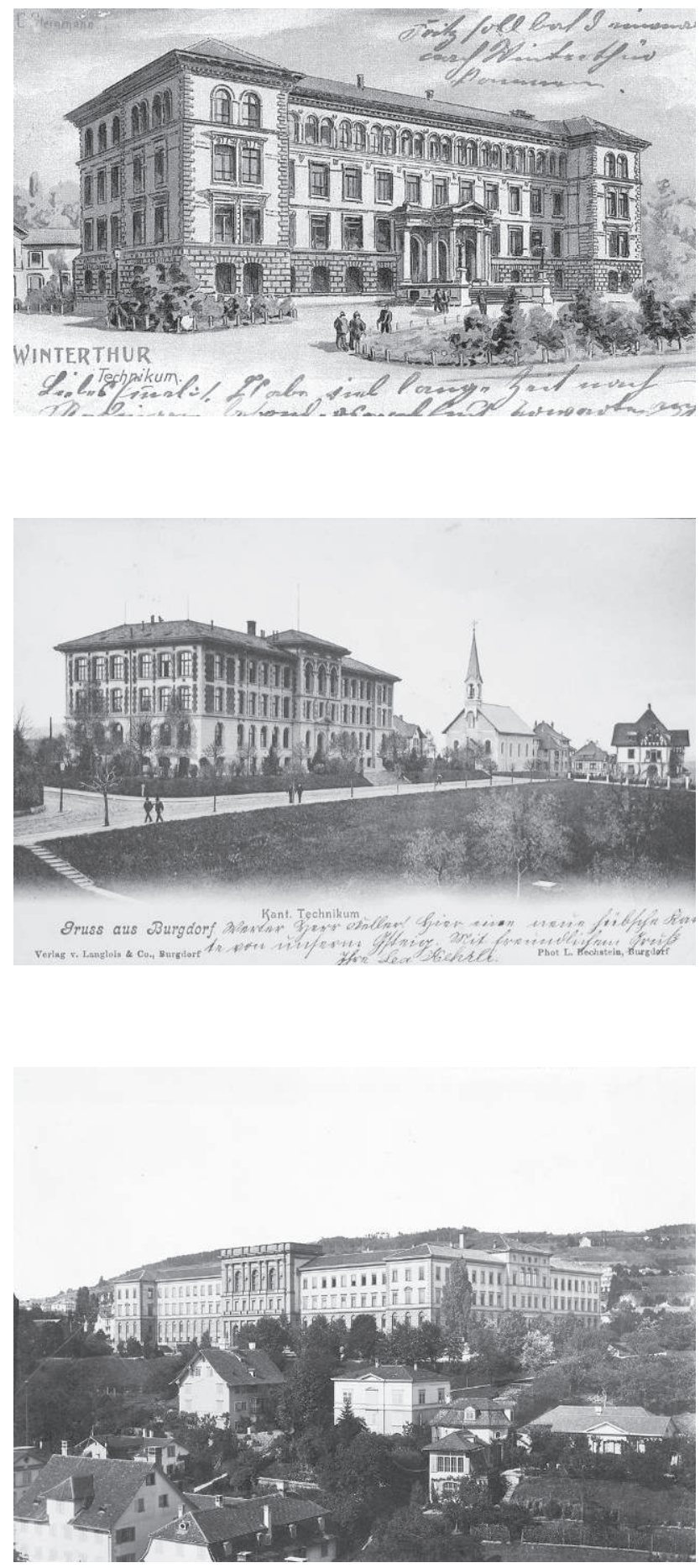

Ill. 4 : Winterthour, technicum.

Carte postale, ca. 1900

Source : Zurich, ETH-Bibliothek, Bildarchiv, Photoglob AG (Zurich), Ans_00827.

Ill. 5 : Berthoud, technicum. Carte postale, s.d. (ca. 1904) Source : Zurich, ETH-Bibliothek, Bildarchiv, Fel_002100-RE.

Ill. 6 : Zurich, École polytechnique fédérale. Photographie anonyme, s.d. (ca. 1870)

Source : Zurich, ETH-Bibliothek, Bildarchiv, Ans_00319. 
suisse Die Eisenbahn (Le chemin de fer), avec élévation et plans : c'est l'un des premiers édifices scolaires à figurer dans les pages du principal journal d'architecture et d'ingénierie suisse. Mais la critique est acerbe : on juge que l'esprit de Semper est dénaturé par des proportions malheureuses et que le style florentin du Cinquecento est trop massif ${ }^{29}$. Ce jugement peu complaisant explique peut-être que cet édifice n'aura pas de descendance : en effet, les technicums de la fin du siècle s'intéresseront à un autre modèle, beaucoup plus prestigieux et plus expressif.

Le premier d'entre eux est construit en 1893-1894 à Berthoud (Burgdorf), chef-lieu de l'Emmental (Ill. 5) ${ }^{30}$. Il est dû aux architectes Otto Dorer et Adolf Füschlin. Le premier avait été auditeur à l'École polytechnique de Zurich puis avait suivi des études à l'École des beaux-arts de Paris. Les deux compatriotes s'étaient ensuite rencontrés dans l'atelier parisien d'Henri Dubois. De retour au pays, Dorer avait ouvert un atelier à Baden en 1884 avant de s'associer à Füschlin en $1890^{31}$. Le technicum de Burgdorf est l'un de leurs premiers gros chantiers. Il propose un plan en E. La façade sur rue, relativement plane, est rythmée par deux avant-corps latéraux et un plus important, central, percé de trois axes de baies. Le rez-de-chaussée surélevé, marqué d'un appareil à refends, supporte deux étages «brique et pierre" percés de larges baies en arc surbaissé; une toiture à l'italienne, à pente faible revêtue de tuiles, couvre le bâtiment. La partie centrale se distingue par sa saillie plus importante par rapport au reste de l'édifice et sa hauteur, par son portail marqué de colonnes engagées et d'un fronton, mais surtout par son premier étage traité en mezzanine servant de piédestal au morceau de bravoure de la façade : les triples baies en plein cintre de la salle centrale du niveau supérieur. La référence saute aux yeux : le schéma en cinq corps, la forme particulière de l'avant-corps central et les éléments italianisants sont autant de références directes à l'École polytechnique de Zurich, l'édifice si célèbre de Semper dans lequel Dorer avait mené ses premières études (Ill. 6). En revanche, le vocabulaire stylistique, l'appareil bichrome, les baies en arc surbaissé, font plutôt référence à l'architecture scolaire française que les deux architectes connaissent bien également. Un

29 Anonyme, "Das Technikum in Winterthur", Die Eisenbahn, vol. 9, n 19,9 novembre 1878, p. 131.

30 Berner Bauten, Berne, Ingenieur- und Architekten-Verein Bern, s.d. [1895].

31 Martin Fröhlich, "Doret, Otto", in DHS : <http://www.hls-dhs-dss.ch/textes/f/F19833.php> (consulté le 12 octobre 2016). 
édifice de synthèse donc : un style évoquant l'architecture scolaire parisienne habille un palais italianisant dans le sillage de l'École polytechnique fédérale.

Une structure similaire - cinq corps, corps central mis en évidence, grandes fenêtres d'une aula dans la partie supérieure - se retrouve à Bienne. Le technicum signé par les architectes Johann Jakob Frey et August Haag est bâti en 1897-1900 sur une éminence dominant la ville, à proximité immédiate de l'école d'horlogerie qui lui sert de premier plan ${ }^{32}$. Son architecture néo-baroque rend la comparaison avec le bâtiment de Zurich moins évidente qu'à Burgdorf, toutefois c'est bien le même schéma qui est utilisé ici (Ill. 2). Dans les deux cas, l'architecture de Semper est citée avec égard et liberté à la fois; elle impose les deux écoles dans le paysage urbain à l'instar de l'École polytechnique qui s'était imposée comme une couronne à Zurich (on parle de Stadtkrone). Et c'est par une réduction des dimensions et des formes - même si à Bienne, le décor est plus riche qu'à Zurich en raison du style baroque choisi - que la déférence au bâtiment-modèle s'exprime, comme si en faisant plus petit et moins monumental, le technicum exprimait la perte du "poly" de son titre et de sa fonction.

Les technicums plus récents suivront d'autres traditions constructives. Celui du Locle (Jean Crivelli, 1901) ${ }^{33}$ joue sur un corps central sur lequel sont branchées deux ailes ressemblant à des ateliers d'horlogerie, industrie dans laquelle la ville est spécialisée (Ill. 7). L'école exprime ici plus la fonction principale de son enseignement qu'une référence savante, sans doute moins pertinente dans cette partie francophone du pays. Quant à l'annexe imposante inaugurée à Winterthour en 1908, due à Hermann Fietz ${ }^{34}$, elle a changé de codes stylistiques, employant un registre régionaliste typique de son temps; mais elle reprend aussi, à vrai dire, les formes des écoles publiques, comme l'édifice initial qu'elle jouxte. Difficile de lire, dans ce duo distant d'une quarantaine d'années, deux écoles professionnelles tant elles ressemblent aux établissements d'enseignement obligatoire qu'elles côtoient.

Les écoles des arts et métiers contemporaines ne s'éloignent guère de l'architecture des technicums et des écoles d'horlogerie, qui semblent avoir joué un rôle conjoint de modèle. À Saint-Gall (Emil Wild, 1886) comme à Zurich (Hermann Fietz, Frauenfachschule [école de couture], 1897, et projet non réalisé

32 INSA, vol. 3, p. 37-39, 99.

33 INSA, vol.6, p. 132-133, 198.

34 Cet architecte zurichois (1896-1931), formé dans des ateliers et auditeur à l'École polytechnique, est l'auteur de plusieurs écoles professionnelles. Voir sa nécrologie dans SBZ, vol.98, 1931, p. 163. 

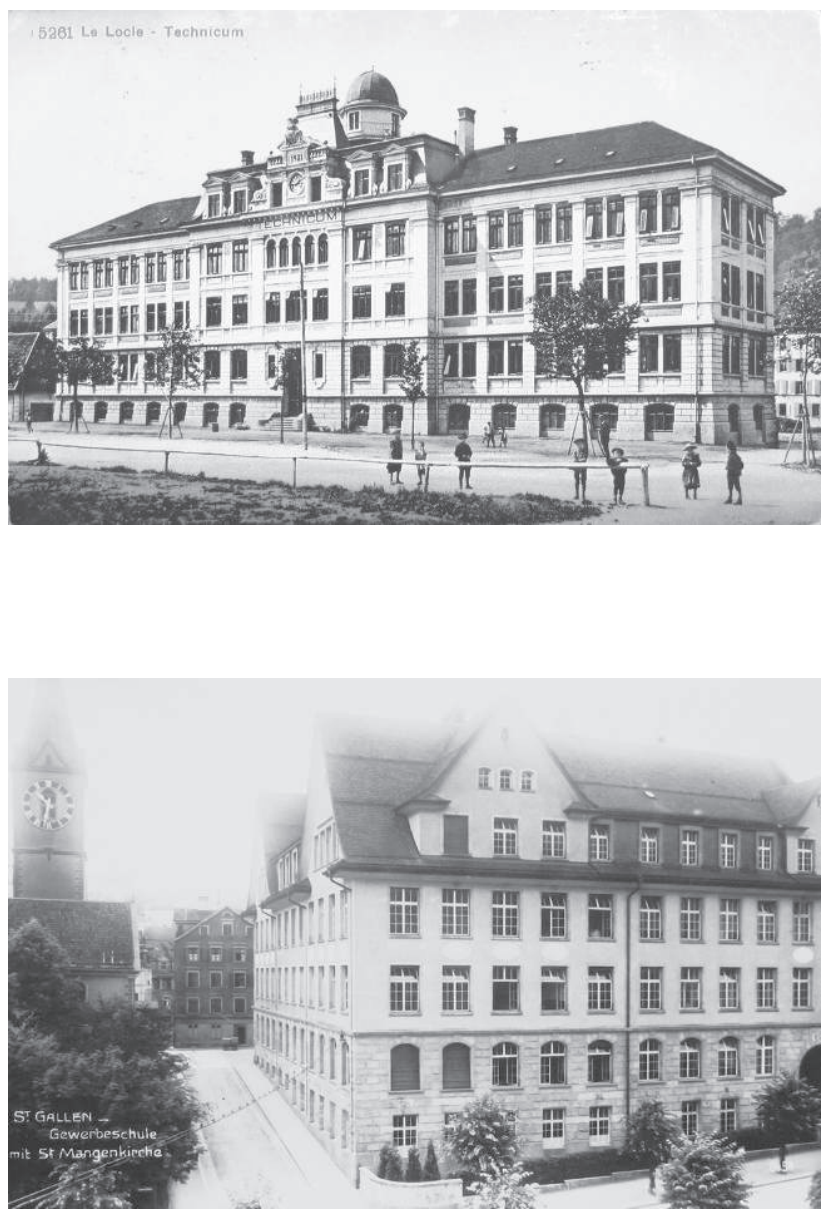

Ill. 7 : Le Locle, technicum. Carte postale, s.d. (ca. 1919)

Source : Zurich, ETHBibliothek, Bildarchiv, Photoglob AG (Zurich), Fel_003178-RE.
Ill. 8 : Saint-Gall, école des métiers. Carte postale, s.d. (ca. 1922) Source : Zurich, ETHBibliothek, Bildarchiv, Fel_005709-RE.

d'Alfred Chiodera, 1900), c'est l'usine et l'école qui sont conjuguées pour créer une architecture parlante ${ }^{35}$. À Aarau (Karl Moser, 1896) et Bâle (Reese et Weber, 1896), dans des contextes urbains particuliers (proximité d'une villa néogothique préexistante; faubourg de la vieille ville, en remplacement du grenier de 1573), l'historicisme sera choisi ${ }^{36}$. Mais à nouveau, dès 1900, l'architecture régionaliste est dominante (Saint-Gall, école des arts et métiers, Paul Truniger, 1912)

35 INSA, vol. 8, p. 167; INSA, vol. 10, p. 361; SBZ, vol.37, 1901, p.68-69 et INSA, vol. 10, p. 228 et 383.

36 SBZ, vol.21, 1893, p.35-36, 42-43, 50-51 et INSA, vol.2, p. 126-128; SBZ, vol.27, 1896, p.8-9 et 15-16; et INSA, vol. 2, p. 192. 
(Ill. 8) ${ }^{37}$, parfois dans le sens d'une architecture commerciale si la fonction de l'école l'appelle (Winterthour, école de la Chambre de commerce, Rittmeyer et Furrer, 1912) ${ }^{38}$.

\section{L'architecture régionaliste comme facteur expressif dans les écoles professionnelles}

On l'a vu, le Heimatstil règle l'apparence d'une majorité d'édifices scolaires en Suisse, à la grande satisfaction d'une partie importante des architectes qui voient dans ce style un équilibre efficace entre tradition, hygiène et exigences du monde moderne. Porté par deux ouvrages de référence dus au Genevois Henry Baudin ${ }^{39}$, le régionalisme connaîtra un succès durable qui en diluera peu à peu la portée idéologique. Toutefois, il faut mettre en évidence un petit ensemble du début du XX $\mathrm{XX}^{\mathrm{e}}$ siècle dont les fonctions semblent particulièrement bien appeler une architecture néo-helvétique : les écoles ménagères et les écoles d'agriculture.

Les écoles ménagères (Koch- und Haushaltungsschule) connaissent un développement notable après l'arrêté fédéral du 20 décembre 1895 sur l'enseignement de l'économie domestique, peu à peu érigée au rang de science sous la triade "ménage, morale et maternité $"^{40}$. Destinées aux jeunes femmes, ces écoles sont souvent aménagées dans des locaux existants, scolaires ${ }^{41}$ ou non ${ }^{42}$. Lorsqu'elles sont édifiées $a d$ hoc, elles obéissent à des codes architecturaux spécifiques répondant à la triade programmatique : simples, donc hygiéniques, les façades porteront des marques patriotiques - puisque c'est aux femmes que reviendra la tâche d'élever les citoyens de demain - et également "féminines".

37 INSA, vol. 8, p. 130.

38 INSA, vol. 10, p. 142.

39 Henry Baudin, Les constructions scolaires en Suisse : écoles enfantines, primaires, secondaires, salles de gymnastique, mobilier, hygiène, décoration, Genève, Éd. d'art et d'architecture, 1907 et Les nouvelles constructions scolaires en Suisse : écoles primaires, secondaires, salles de gymnastique, hygiène, décoration, Genève, Éd. d'art et d'architecture, Kundig/Paris, H. Gaulon, 1917.

40 Tamlin Schibler, Fées du logis : l'enseignement ménager dans le Canton de Vaud de 1834 à 1984 , Lausanne, Bibliothèque historique vaudoise, 2008, p. 52.

41 Par exemple à Lausanne dans le socle de l'École moyenne de Saint-Roch, bâtie en 1874 .

42 En Suisse alémaniques, des villas bourgeoises sont transformées en écoles ménagères après la Première Guerre mondiale. C'est le cas plus largement pour les écoles féminines : à Zurich, l'École d'art pour femmes est installée de 1899 à 1913 dans la villa du peintre Arnold Böcklin, édifiée en 1885 (INSA 10, p. 317). 
L'écoles de Berne (Christian Trachsel, 1902) ${ }^{43}$ use ainsi de formes domestiques, qui l'assimilent aux immeubles de rapport de son époque : façade asymétrique, fenêtres en arc surbaissé, balcons de bois, toiture à la mansart percée de lucarnes pittoresques sont autant de marques bien peu expressives pour une école des métiers mais qui peuvent évoquer la tranquillité et le bien-être d'un chez soi (Heim) chaleureux. À Zurich (Robert Zollinger, 1909), une partie de ces caractéristiques est reprise mais le rythme monumental imposé par les travées régulières marquées par des lésènes (soit des pilastres sans base ni chapiteau) confère un caractère davantage public à l'édifice ${ }^{44}$.

L'équivalent masculin est représenté par les écoles d'agriculture qui se multiplient au début du XX $\mathrm{XX}^{\mathrm{e}}$ siècle à la suite de la loi fédérale de 1893 sur l'agriculture, qui prévoit un soutien financier aux cantons possédant de telles écoles. Forcément situées en campagne, elles seront souvent liées à des écoles ménagères rurales, pendant des écoles d'économie domestique urbaines qui forment les femmes à la paysannerie moderne ${ }^{45}$. Dès son origine, l'École polytechnique de Zurich possédait une section agricole, mais elle avait été rapidement désertée au profit des écoles cantonales qui, pour certaines, avaient été créées dès les années $1850^{46}$. Les premières écoles s'installent souvent dans des édifices préexistants, sans grandes prétentions architecturales. Ce n'est qu'au sortir de la Grande Guerre que des édifices spécifiques sont réalisés dans plusieurs cantons, avec un soin tout particulier. À Marcelin, près de Morges (1921-1923), Georges Epitaux imagine un vaste ensemble d'édifices qui abrite les deux écoles agricole et ménagère dans des ailes distinctes. Leur architecture appartient à la seconde période du Heimatstil, parfois qualifié d'Heimatstil "objectif" puisqu'il ne cherche plus une lecture historiciste de l'architecture traditionnelle (Ill. 9). Régularité, façades blanches et lisses, grandes toitures

43 INSA, vol. 2, p. 480.

44 INSA, vol. 10, p. 433.

45 Tamlin Schibler, Fées du logis..., op. cit., p. 53.

46 Ce sujet demeure complètement inexploré. Après une brève recherche à partir notamment des ouvrages célébrant les anniversaires des écoles (catalogue de la Bibliothèque nationale, www.helveticat.ch), on peut citer comme principales écoles (simples, ou doubles - agriculture/ménagère rurale - signalées par un astérisque; date de fondation, pas forcément de construction d'une école) : Zurich-Strickhof (1853), Rütti (1860), Lausanne (1870) puis Marcelin (1922), Sursee (1885), Cernier (1885); après la loi fédérale de 1893 : Grangeneuve* (1898), Arenenberg (1904), Schwand-Münsingen* (1909), Wetzikon (1911), Affoltern (1912), Waldhof* (1920), Willisau (1921 ; école ménagère et formation de la paysanne dès 1946), Sion-Châteauneuf (1923), Winterthur-Wülflingen* (Weiland, 1927), Courtemelon* (1927), Flawil (1932), Uster* (1949), Langau-Bäregg (1958). 

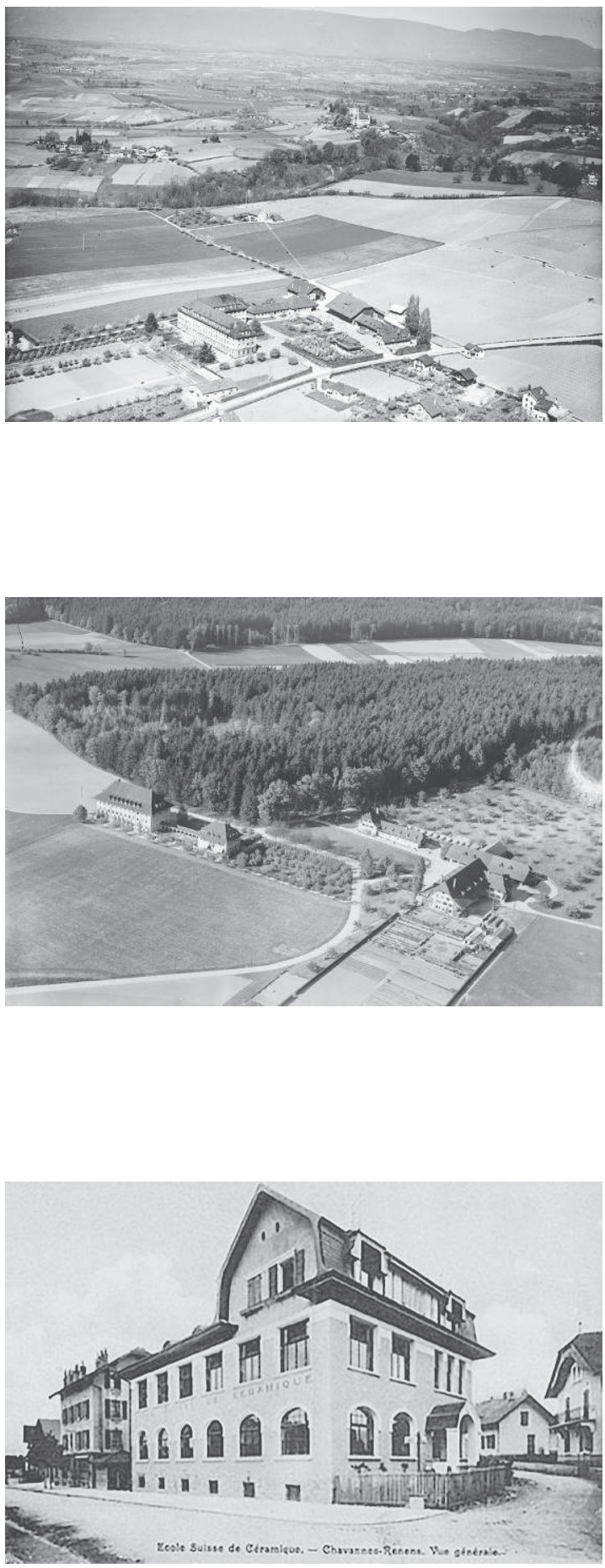

Ill. 9 : Morges, école d'agriculture de Marcelin. Photographie aérienne de Werner Friedli, 1940 Source : Zurich, ETH-Bibliothek, Bildarchiv, Stiftung Luftbild Schweiz, LBS_H1-011729 / CC BY-SA 4.0.
Ill. 10 : Langenthal, école d'agriculture de Waldhof. Photographie aérienne de Werner Friedli, 1953

Source : Zurich, ETH-Bibliothek, Bildarchiv, Stiftung Luftbild Schweiz, LBS_H1-014801 / CC BY-SA 4.0.
Ill. 11 : Chavannes-près-Renens, école de céramique.

Carte postale, s.d. (ca. 1914) Source : coll. part. 
à croupes suffisent à donner du caractère à cette école dont le plan en L ne s'éloigne guère des grands collèges du temps, tant en volume qu'en distribution. À partir d'une cage d'escalier formant vestibule et placée à l'intersection des deux ailes, côté cour, des couloirs et les salles communes principales (réfectoire, salle des collections) donnent accès aux salles d'enseignement tournées vers le sud et l'ouest. De vastes dépendances aux formes agrestes permettent aux élèves de se familiariser avec le travail agricole.

L'école conçue par Michel Polak et Alphonse de Kalbermatten à Châteauneuf près de Sion (1922-1923) est dans la même veine, tout comme celles de Prince et Béguin à Cernier (1922), Hector Egger à Langenthal (1923) (Ill. 10) et Hermann Fietz à Winterthour (1927). Visiblement, ces écoles se réfèrent peu ou prou aux résidences campagnardes du patriciat de l'Ancien Régime, quand ce n'est pas à l'architecture conventuelle de l'époque baroque, si sobre parfois en Suisse. Le rôle joué par le style est ici clair et, à nouveau, idéologique. Dans une Suisse déchirée entre une certaine forme de modernité et un retour aux valeurs patriotiques qui culminera durant la Seconde Guerre mondiale, l'anoblissement de l'agriculture au rang de science enseignable par une architecture sobre mais connotée socialement, n'est pas un hasard.

D'autres types d'écoles professionnelles font aussi recours au régionalisme. On peut ainsi citer l'École de céramique de Chavannes-près-Renens (Eugène d'Okolsky, 1912), qui ne se différencie guère de l'École d'horlogerie de la Vallée de Joux, déjà évoquée, ou de certaines écoles ménagères alémaniques (Ill. 11). Les cartes sont brouillées.

\section{La loi de 1930 et ses effets}

La pénurie de main-d'œuvre qualifiée conjuguée à la xénophobie ambiante - en plein chômage, dans les années 1920-1923, on se plaint de devoir engager des maçons étrangers et notamment des contremaîtres qu'il faudra mieux payer que les manœuvres helvétiques ${ }^{47}$ - engendre la rédaction d'une loi fédérale sur la formation professionnelle, perçue comme le seul moyen d'assurer une diminution du chômage et une ascension sociale aux citoyens suisses. Initiées à la fin de la Grande Guerre, les discussions prennent fin en 1930, date de son adoption par les autorités fédérales. La loi fixe les formes de l'apprentissage 
professionnel et, surtout, le normalise par un examen de maîtrise; charge aux cantons d'organiser les cours et de fonder les écoles ad hoc ${ }^{48}$. Dans les années 1930, en dépit de la loi, la situation est toutefois loin d'être homogène. Deux systèmes coexistent : dans la partie germanophone prime le Dualsystem (apprentissage en entreprise, cours dans une école professionnelle); dans les parties francophone et italophone sont en revanche privilégiées les écoles des métiers qui forment les apprentis dans 37 professions différentes. La répartition géographique de ces écoles des métiers prouve bien cette prédilection latine ${ }^{49}$ : on en dénombre alors 40 dans l'ensemble de la Suisse, 20 en terres alémaniques, 20 en Suisse romande et au Tessin, ce qui d'un point de vue démographique est tout à fait disproportionné50. La majorité des écoles se trouve encore en terres réformées (28), traditionnellement plus scolarisées, les cantons catholiques (et ruraux) demeurant largement sous-dotés (12 ${ }^{51}$. Pour faire évoluer cette situation, l'état fédéral soutien la construction d'écoles là où elles manquent par des subventions plafonnées à $20 \%$ du coût total de l'édifice, au maximum 200000 francs $^{52}$.

En dépit d'une période morose du point de vue économique - les effets du krach boursier de Wall Street se font ressentir à partir de 1931 en Suisse -, plusieurs chantiers sont lancés grâce à un soutien fédéral, en particulier dans des villes de moyenne importance (Baden, Langentahl) mais aussi dans des chefs-lieux cantonaux (Lausanne, Zurich) ${ }^{53}$. De manière générale en Suisse, cette décennie se caractérise par une architecture tentée par l'avant-garde, que l'historiographie a qualifiée de Neues Bauen. Plusieurs des écoles en question se rattachent de manière évidente à ce courant, notamment à Baden (école des firmes $\mathrm{ABB}$ et $\mathrm{BBC}^{54}$, par le bureau des constructions $\mathrm{BBC}$, 1931; école des arts et métiers, A. Gantner, 1932) ${ }^{55}$, Zurich (école des arts et métiers et musée,

48 Loi fédérale du 26 juin 1930, art. 29 et 30.

49 Feuille fédérale, 25 septembre 1984, n³8, p. 1407, tableau des écoles à la fin des années 1930.

50 En 1930, sur un total de 4041602 habitants, $72 \%$ de la population helvétique est germanophone, 20,5 \% francophone, 5,9\% italophone, $1 \%$ rhéto-romanche, 0,6 \% allophone. Cf. Historical statistics of Switzerland online :<http://www.fsw.uzh.ch/hstat> (consulté le 20 octobre 2016).

51 On compte alors 9 cantons protestants, 4 cantons biconfessionnels (à majorité réformée) et 12 cantons catholiques.

52 Loi fédérale du 26 juin 1930, art. 50 al. e et art. 51 al. e.

53 Un projet existe pour Bâle, dû à Hermann Baur; il sera réalisé ultérieurement. Cf. Das Werk, vol. 30, 1943, p. 191-192 et vol. 49, 1962, p.61-70.

54 ABB : Asea Brown Boveri; BBC : Brown, Boveri \& Cie.

55 Neues Bauen in der Schweiz, op. cit., vol. 2, p. 24-25. 
Steger et Egender, 1933) ${ }^{56}$ (Ill. 12) et, dans une moindre mesure, Lausanne (Dubois, Favarger, Godet et Gilliard, 1930). Les écoles alémaniques déclinent les caractéristiques du Neues Bauen - plan asymétrique, toiture plate, façades pâles et nues percées de longues fenêtres d'inspiration industrielle -, alors que celle de Lausanne fait preuve d'un certain formalisme (composition symétrique, jeu sur la modénature), sans doute dû à des architectes moins audacieux (Ill. 13). Dans ce dernier cas, l'histoire complexe de l'édifice explique aussi en partie la modernité retenue des formes. Ainsi, la toiture plate, protégée par des murets et des parois en brique de verre, est-elle aménagée en fin de chantier à la place de la toiture en tuiles prévues initialement, afin de pouvoir pratiquer des enseignements en plein air ${ }^{57}$. Quant à la structure en béton armée permettant de larges plateaux sans autres séparations que quelques piliers, elle est pensée pour permettre une occupation souple des espaces. En revanche, la cage d'escalier monumentale au centre de l'édifice implique une contrainte de distribution dont l'origine n'est pas liée au programme mais bien à la forme symétrique de l'édifice. L'inspiration formelle est ici très clairement affirmée, comme le soulignent les architectes au moment de l'inauguration : "Tout a été traité, à l'intérieur comme à l'extérieur, avec économie, en laissant à l'école le caractère qu'elle doit avoir franchement : celui d'une usine $n^{58}$.

Assez rapidement toutefois, cette ouverture architecturale fait place aux formes renouvelées du régionalisme, le Landistil, du nom de l'Exposition nationale suisse de Zurich (Landi, 193959) qui avait contribué à leur large et rapide diffusion. Reprenant certains éléments du Neues Bauen - notamment l'asymétrie des plans et des partis, l'usage de techniques modernes de construction, les façades sobres largement percées, l'intégration d'œuvres d'art au bâti (le Kunst am Bau) -, le Landistil les rend moins modernes par le recours aux toitures à deux pans en tuiles, à des matériaux plus traditionnels (notamment l'usage du bois pour les huisseries, toujours laissé visible) et l'ajout de motifs (arcades, colonnes de pierre apparente, etc.) remontant en fait au Heimatstil. Plusieurs écoles professionnelles des années 1940-1950 font usage de cette expression, notamment celles édifiées en campagne (Langenthal, Gewerbeschulhaus, Hector

56 GAS 1, p. 171, INSA, nº 10, p. 302.

57 À ce sujet, voir Dave Lüthi, "L'école-usine...", art. cit.

58 Das Werk, vol. 4, 1933, p. 110.

59 Diminutif de Landesausstellung (exposition nationale). 

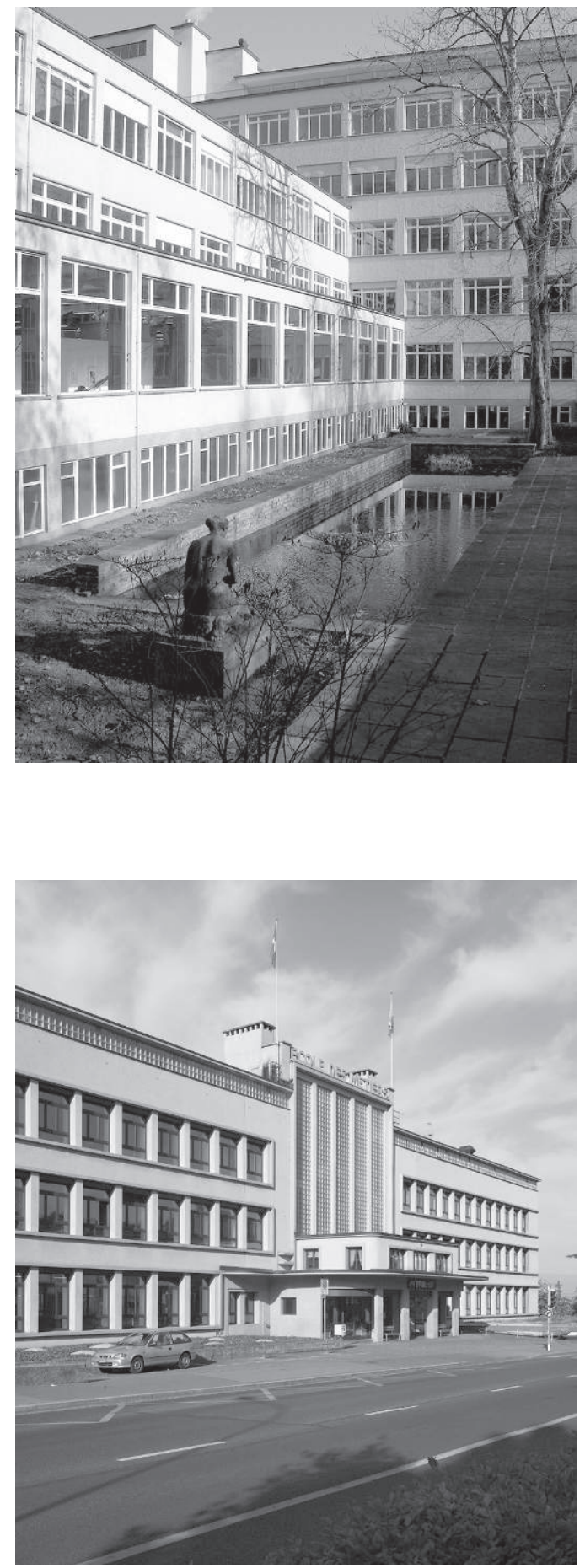

Ill. 12 : Zurich, école des arts et métiers

Photographie Dave Lüthi, 2008.
Ill. 13 : Lausanne, école des métiers () Davide Quattrocchi, 2011. 
Egger, 1938; Kaufmännische Berufsschule, Walter Schwaar, 1951). Mais à nouveau, cette architecture ne dénote plus la fonction de l'édifice : l'essentiel des écoles de cette époque, primaires et secondaires notamment, sont également bâties dans le Landistil, qui remplace en le modernisant un peu le Heimatstil dans l'ambiance protectionniste de l'immédiat après-guerre.

En dépit de ce contexte crispé, une série d'établissements se réfère à l'un des plus célèbres et des plus modernes des années 1930 : l'École des arts et métiers de Berne (1937-1939), due à Hans Brechbühler et très largement diffusée dans les revues d'architectures (Ill. 14) ${ }^{60}$. Cet ancien collaborateur de Le Corbusier à Paris ${ }^{61}$ avait bien retenu les leçons du maître, notamment les Cinq points de l'architecture moderne, en concevant cet édifice majeur du paysage urbain bernois. En volume, en coupe comme en élévation, le bâtiment doit beaucoup aux projets contemporains de Le Corbusier; la trame de façade, en particulier, fait référence à la Cité de refuge de l'Armée du Salut à Paris (1929-1933) dont Brechbühler a pu suivre la construction. Les ateliers sont traités comme un corps indépendant, coiffé d'une verrière au caractère industriel. À sa suite, mais bien plus tardivement, l'École professionnelle de Lausanne (Brugger, Perrelet, Stalé, Quillet, 1953-1955) fera en particulier une référence explicite à la fois à l'œuvre de Le Corbusier ainsi qu'à l'école bernoise, sans que le lien soit cette fois direct - sinon par le fait que Frédéric Brugger a travaillé vers 1940 dans les bureaux d'Otto Salvisberg et d'Hermann Baur, autres chantres de la modernité helvétique et contemporains de Brechbühler (Ill. 15) ${ }^{62}$.

L'architecture scolaire suisse reste frappée très longtemps par l'esthétique corbuséenne ${ }^{63}$, parfois dans une version très amenuisée, comme à l'école professionnelle d'Olten (Bitterli, Barth, Zaugg), avec sa toiture plate débordante, typique de l'architecture des années 1950, et avec ses façades modulaires qui doivent autant - si ce n'est plus - à Brechbühler qu'à Le Corbusier ${ }^{64}$. Plus tard, à Bâle (Gewerbeschule, Hermann \& Peter Baur, Bräuning, Dürig, 1956-1961), si

60 Voir notamment la série d'articles consacrée à cet édifice dans Das Werk, 1938, vol. 5, 1940, vol.7, 1941, vol. 5 .

61 Brechbühler fréquente Le Corbusier en 1930-1931. Cf. Ueli Zbinden (dir.), Hans Brechbühler 19071989, Zürich, Gta Verlag, 1991, p. 10-14 et 30-59.

62 Bruno Marchand, "Souvenirs intenses. Hommage à Frédéric Brugger (1912-1999)", Habitation, 1999, p. 20.

63 Bruno Corthésy, Bruno Santos, "Le Corbusier à Lausanne. L'architecte Jacques Dumas, le cas d'un émule corbuséen. Le Centre universitaire catholique, la chapelle du Servan et l'école du Riolet à Lausanne", Monuments vaudois, $\mathrm{n}^{\circ}$ 7, 2017, p. 56-66.

64 Il faudrait à cet égard s'intéresser de plus près au rôle de Brechbühler comme enseignant à l'École 

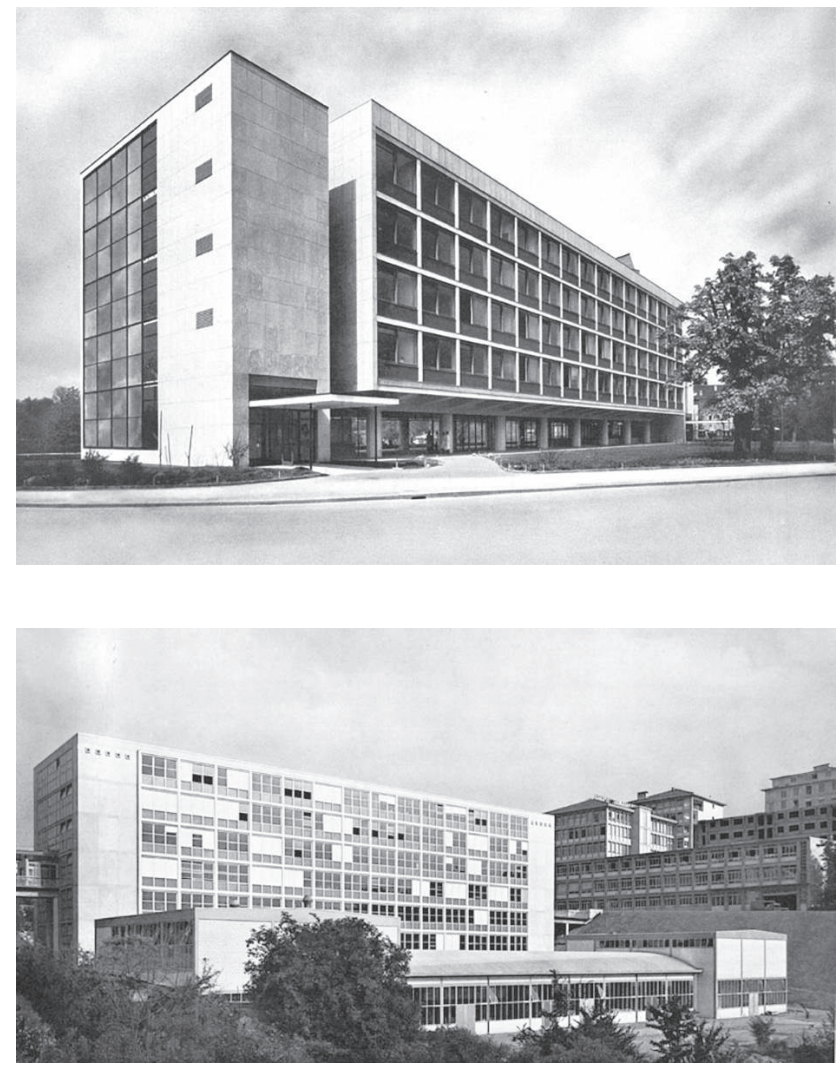

Ill. 14 : Berne, école des arts et métiers Source : Werk, bauen und Wohnen.

Ill. 15 : Lausanne, école professionnelle Source : Les écoles professionnelles complémentaires, Lausanne, s.n., 1955.

le plan en peigne d'un projet inabouti de 1943 dû à un autre émule du maître, Hermann Baur, n'est pas modifié, l'esthétique corbuséenne de l'édifice évolue avec son temps ${ }^{65}$. Comme Brechbühler, Baur s'inspirait en 1943 des édifices corbuséens du début des années 1930; la version des années 1950 de son projet montre sa connaissance du développement de la carrière de son maitre à construire : certains traits - notamment le traitement sculptural des formes traitées en béton ainsi que la distribution à partir d'un couloir longitudinal doivent beaucoup aux Unités d'habitation. Le parti de l'immeuble corbuséen

polytechnique de l'université de Lausanne dès 1956. Sur ce point, voir Kurt Aellen, "Die Lehre Hans Brechbülers ", in Ueli Zbinden (dir.), Hans Brechbühler 1907-1989, op. cit., p. 158-159.

65 Hermann Baur, "Neubau der Allgemeinen Gewerbeschule Basel 1956-1961 ", Das Werk, 1962, vol. 2, p. 61-70; Rebekka Brandenbeger, "Ordnung in Freiheit. Beton und Denkmalpflege", Art + Architecture en Suisse, 2009/4, p. 28-35. 
perdurera des décennies encore ${ }^{66}$, mais habillé parfois, on va le voir, dans des drapés qui ne doivent plus rien au Français d'origine helvétique.

\section{Renouveau de l'enseignement, américanisation de l'architecture}

Les modifications du marché de l'emploi de l'après-guerre (industrialisation de la société au détriment de l'artisanat, qui appelle des ouvriers mieux formés ${ }^{67}$ ) doublées de la crainte tenace d'un envahissement par la main-d'œuvre étrangère, impliquent en 1963 une refonte complète de la loi de 1930, fondée sur des paramètres complètement différents. Entre temps, le nombre d'apprentis a plus que doublé et, dorénavant, 238 professions sont enseignées ${ }^{68}$. Cette ouverture de la formation professionnelle qui fait sans doute écho à la démocratisation contemporaine des études supérieures, s'accompagne aussi d'autres types de prises de conscience. L'apprenti est peu à peu considéré comme une personne à part entière, dotée de capacités professionnelles spécifiques, dont le choix de carrière influe sur la vie tout entière; on réalise aussi que les travailleurs peuvent changer de profession durant leur vie, ce qui implique le développement de la formation continue, notamment pour les chômeurs. Les subventions fédérales sont considérablement augmentées, renforçant donc le rôle de l'État dans des écoles pourtant toujours gérées par les cantons. De fait, leur construction connaît une véritable explosion, tant dans les chefs-lieux cantonaux que dans les villes de moindre importance.

Cette mutation de l'enseignement professionnel provoque celle de son architecture. Alors même qu'aucun programme ou modèle fédéral n'est connu ${ }^{69}$, un type s'impose durant près de deux décennies : celui élaboré dans les années 1940 déjà par Ludwig Mies van der Rohe dans le cadre universitaire, notamment à l'Illinois Institute of Technology de Chicago (1943-1957). À défaut de bien

66 Notamment : Lugano Trevano, 1973-1975 et Lenzbourg, Gewerbeschule, 1978. Cf. GAS 3, p. 282; "Gewerbeschule Lenzburg", SBZ, vol.96/38, 1978, p. 721-726.

$6795 \%$ des ouvriers sont formés dans de grandes entreprises, $5 \%$ dans des ateliers ou des écoles d'arts appliqués. Voir Jean-Pierre Tabin, Formation professionnelle..., op. cit., p. 112.

68 Ibid.

69 Il faudrait toutefois le vérifier par des études de cas, ce qui n'est pas possible dans le cadre de cet article. 
saisir la chronologie et les enjeux du remploi de sa pratique en Suisse $^{70}$, on peut constater que cette architecture se superpose puis supplante celle de Le Corbusier dès les années 1960. Ce dernier, perçu comme lyrique et poète, a le défaut de "négliger la matière, la technique». En revanche,

"[Mies], architecte "anatomique”, établit une hiérarchie d'ordre constructif; il distingue nettement la structure primaire, soit l'ossature portante (en fer ou en béton) de la structure secondaire, soit les parois de remplissage (verre, brique, etc.). Il recherche la simplicité et la perfection ultime de l'exécution technique. Il met en valeur la noblesse naturelle des matériaux qui s'affirment en grands panneaux unis. Les proportions sont d'une rigueur classique; tout est équilibre, élégance, pureté, symétrie même ${ }^{71}$.

Autant de qualités qui expliquent sans doute la véritable fascination exercée par l'Allemand américanisé sur toute une frange de jeunes architectes suisses, dont certains partent découvrir son architecture et visiter son atelier à New-York ${ }^{72}$.

Cette passion pour l'architecture réglée et "minimaliste" de Mies s'explique sans doute par la rationalisation de l'architecture suisse des Trente Glorieuses. Le mouvement était né comme ailleurs durant l'entre-deux-guerres - même si en Suisse il n'était pas motivé par la reconstruction, mais par la crise économique -, mais il connaît un essor sensible sous l'influence grandissante des ingénieurs qui se muent parfois en architectes. La construction scolaire devient un véritable laboratoire, les démographes prédisant une croissance importante du nombre d'élèves pour les vingt ans à venir, croissance qui sera relativisée après le choc pétrolier et la crise horlogère des années 1970. La question de la standardisation est au centre de la réflexion, notamment pour les matériaux et les structures ${ }^{73}$, donnant lieu, entre autres, à la création du système de construction CROCS, mis au point à Lausanne pour les écoles communales et employé largement en Suisse romande ainsi qu'à l'étranger ${ }^{74}$. De 1964 à 1977, ce ne sont pas moins de huit écoles professionnelles qui empruntent à la manière

70 Pour le moment, voir : Bruno Corthésy, "La tour des Imprimeries réunies lausannoises : la permanence du modèle miessien", Art + Architecture en Suisse, vol. 58/1, 2007, p. 39-45.

71 Paul Waltenspühl, "Tendances de l'architecture contemporaine», Habitation, vol. 26, 1954, p. 18-19, ici p. 18.

72 Bruno Corthésy, art. cit., p. 40-41.

73 Peter P. Schweger, "Système de construction pour centres d'éducation", Bauen + Wohnen, vol. 19, 1965, p. 269-247.

74 À ce sujet, voir Aline Suillot, "Les CROCS : "construire mieux, plus vite et moins cher" ", in Dave Lüthi (dir.), Lausanne-les écoles, op. cit., p. 110-121. 
miessienne, réparties sur le plateau suisse d'Yverdon à Zurich, avec un élément isolé à Sion, en Valais ${ }^{75}$. La haute école professionnelle de Windisch, due à Fritz Haller (1964-1968), est l'une des plus abouties, et sans doute la plus significative en raison de la notoriété de son auteur ${ }^{76}$ (Ill. 16). Elle reprend nombre de caractéristiques de l'architecture de Mies, tant en volumétrie (trois blocs carrés et rectangle posés régulièrement dans un parc), en distribution (salles disposées autour d'un hall central) qu'en élévation (ossature en acier, remplissage en verre filtrant stratifié, profils en acier inoxydable) (Ill. 17). Membre de l'École de Soleure, Haller s'intéresse depuis le début des années 1960 à la construction modulaire en acier, dont il fait sa marque de fabrique - on lui doit la célèbre ligne de mobilier modulable USM (1963) ${ }^{77}$. À l'époque du chantier de Windisch, il travaille à la University of Southern California comme collaborateur libre à l'Institute for Building Research de Konrad Wachsmann, un ancien collaborateur de Le Corbusier et de Walter Gropius, auprès duquel il a aussi pu s'intéresser à la production de Mies. Le catalogue de ses œuvres prouve cependant que le modèle miessien ne se restreint pas aux écoles professionnelles, suivant encore en cela les principes de Mies sur la neutralité des formes ${ }^{78}$. D'autres exemples célèbrent avec une certaine rigueur le modèle américain. Le centre d'enseignement d'Yverdon (Frédéric Brugger, 1965-1967) juxtapose plusieurs corps dont un en forme d'usine (sheds en toiture, cheminée) tandis que sa blancheur tranche avec le graphisme du corps principal qui met en valeur la structure en acier $^{79}$. Les écoles des métiers de Bienne (Alain Tschumi, 1973-1975) et de Lucern-Horw (Peter Stutz, 1970-1977) confinent elles au pastiche ${ }^{80}$.

Le lien entre l'architecture des écoles professionnelles et le recours à des techniques constructives modernes s'explique aisément par la réflexivité qui

75 Cette école est moins mimétique que les autres, sans doute, mais c'est la plus précoce du corpus (Paul Morisod, Jean Kyburz, 1960-1964). Cf. GAS, vol. 3, p. 217; Christophe Valentini (dir.), L'architecture du 20 siècle en Valais, 1920-1975, Golion, Infolio, 2014, p. 161.

76 Laurent Stalder (éd.), Fritz Haller : Architekt und Forscher, Zurich, Gta Verlag, 2015, p. $276-281$.

77 Jürg Graser, Gefüllte Leere. Das Bauen der Schule von Solothurn: Barth, Zaugg, Schlup, Füeg, Haller, Zurich, Gta Verlag, 2014.

78 Notamment l'école cantonale de Baden (1957-1964) et plusieurs projets de concours (Laurent Stalder (dir.), Fritz Haller..., op. cit., 2015, p.264-267; dans le même ouvrage : Bruno Maurer, "Hallers Traum. Die Schule als "allseitig offene System" ", p. 54-77).

79 GAS, vol.3, p. 85.

80 "Gewerbeschule Biel», Das Werk, vol.63, n6, 1976, p. 396-402 et GAS, vol. 2, p. 225; Heinz Krehl, "Zentralschweizerisches Technikum Luzern in Horw : ein notwendig kritischer Bericht", Bauen + Wohnen, vol.24/8, 1970, non paginé). 

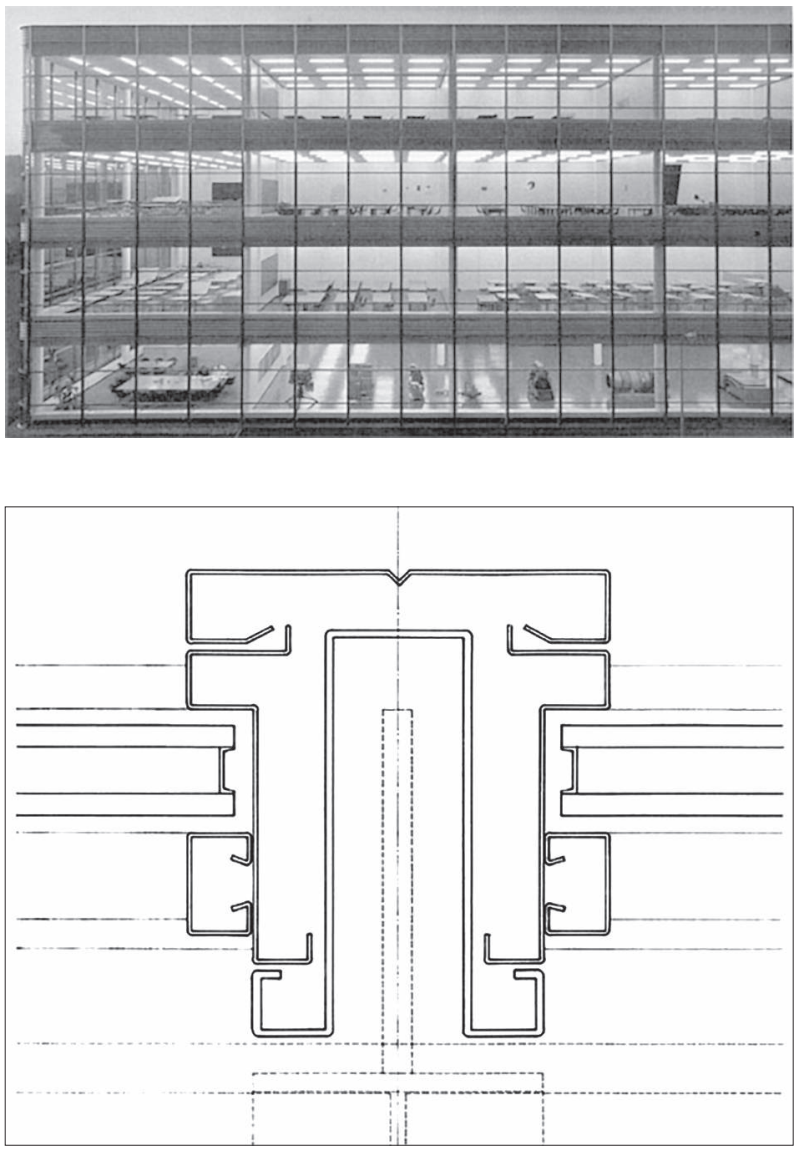

Ill. 16 : Windisch, école technique supérieure, corps principal Source : Bauen + Wohnen, 1968.

Ill. 17 : Windisch, école technique supérieure. Coupe d'une traverse verticale de la paroi extérieure en tôle d'acier au nickel chromée repliée

Source : Bauen + Wohnen, 1968.

s'établit entre les cours dispensés et l'architecture environnante. Sans reproduire exactement le principe de l'usine-école parfois décliné précédemment, ce n'est sans doute pas un hasard si ces écoles s'intéressent aussi tôt à une architecture qui ne deviendra commune que dans les années 1970. À cette époque, plus aucun programme n'échappe à l'architecture miessienne : les universités ne seront d'ailleurs pas les dernières à s'y convertir, signe que le caractère "parlant" qu'on a pu mettre en évidence dans les écoles professionnelles n'est pas une règle générale. Il s'agit avant tout d'un effet de mode, mais aussi de réponse à des problèmes concrets, économiques, démographiques et idéologiques.

Contre toute attente, quelques édifices tentent le mariage des deux tendances majeures du XX ${ }^{\mathrm{e}}$ siècle en matière d'architecture : Le Corbusier et Mies van der Rohe. Si le plan et la volumétrie demeurent fréquemment corbuséens, 
sur le modèle plus ou moins assumé de l'école des métiers de Berne, les façades modulaires empruntent volontiers formes, matériaux et expression au vocabulaire miessien. L'école des arts et métiers de Lenzbourg (Zimmerli \& Blattner, 1978 ${ }^{81}$ en est un bon exemple, de même que celle de Winterthour $\left(\right.$ Peter Stutz, 1974) ${ }^{82}$, mais dans une moindre mesure en raison de la structure tridimensionnelle de la façade (brise-soleil) en acier Cor-Ten qui perturbe la lecture du bloc lisse et sculptural au profit d'une écriture plus maniériste et pittoresque, si l'on ose dire, de l'épiderme. Stutz connaît peut-être les réalisations d'Egon Eiermann en Allemagne, notamment le centre administratif Olivetti à Francfort-sur-le-Main (1968-1972), doté d'un système de double façade en filigrane d'acier demeuré célèbre ${ }^{83}$. On notera que l'alliance inverse - plan carré miessien allié à des façades plus corbuséennes - existe aussi, mais plutôt dans le domaine des hautes écoles ${ }^{84}$, ce qui pourrait confirmer l'hypothèse de l'emploi d'une architecture "technique" en particulier là où elle a du sens. Enfin, il faut mentionner une autre curiosité : l'école des métiers de Heimbach à Lucerne (Josef Gasser, Gottfried Wielandt, 1956-1958) qui allie une esthétique corbuséenne à une structure qui s'inspire des expériences de Wright pour le plan et la structure en pyramide inversée ${ }^{85}$.

\section{Un éclectisme final, ou un manque de recul historique?}

La loi fédérale de 1978 va modifier, une fois encore, les écoles professionnelles en les spécialisant davantage (les enseignants doivent dorénavant être formés à la pédagogie) ${ }^{86}$; elle annonce la révision fondamentale de 1995 qui les transforme en sept hautes écoles spécialisées (HES) réparties sur le territoire national.

81 "Gewerbeschule Lenzburg", SBZ, vol.96/38, 1978, p.721-726.

82 "Der Neubau der Gewerblichen Berufsschule Winterthur», Das Werk, vol.63, 1976, p.522-525; GAS, vol. 1, p. 150.

83 Lors de voyages aux États-Unis, Eiermann avait rencontré Marcel Breuer, Gropius et Mies van der Rohe (Immo Boyken, Hans J. Oestmann, "Ludwig Mies van der Rohe and Egon Eiermann: The Dictate of Order ", Journal of the Society of Architectural Historians, vol.49/2, 1990, p. 133-153.

84 Sur le modèle notamment de l'école cantonale de Freudenberg à Zurich (Jacques Schader, 19591960) : école cantonale de Winterthour (Erik Lanter, 1960-1963) et Lausanne, collège secondaire et école d'art de l'Élysée (Frédéric Brugger, 1961-1964) et pensionnat de jeunes filles (Max Richter et Marcel Gut, 1961-1964). Cf. GAS, vol. 1, p. 196 et 145; GAS, vol. 1, p. 109.

85 GAS, vol. 1, p. 264.

86 Jean-Pierre Tabin, Formation professionnelle..., op. cit., p. 127. 
À cette période de mutation semble correspondre une période de recherche architecturale qui n'est peut-être qu'un effet d'optique, le corpus devenant de plus en plus difficile à cerner. Notons tout de même que les principales tendances du temps - du brutalisme au post-modernisme - sont représentées. Il est difficile de repérer des courants forts : à côté des édifices qui s'inscrivent encore dans les tendances corbuséenne ou miessienne ${ }^{87}$, on observe un goût marqué pour le béton armé, inauguré par la célèbre halle des maçons de Baur à Bâle, dont témoignent à leur manière les sheds "industriels" de Heidi et Peter Wenger à Changins (école d'agriculture et centre de recherche, 1973-1975) ${ }^{88}$ qu'on retrouve sous d'autres formes et structures à l'École de la construction de Tolochenaz (1985-1988) où Patrick Mestelan et Bernard Gachet déclinent un plan régulier, symétrique, hiérarchisé, qui doit autant au post-modernisme de l'école tessinoise qu'aux réalisations plus anciennes de Louis Kahn, dont la vogue est alors sensible en Suisse romande ${ }^{89}$.

À défaut de pouvoir évaluer l'évolution récente de l'architecture des écoles professionnelles, on peut tenter une synthèse des tendances historiques. Aux premières écoles, souvent privées et patronales, inspirées par les usines (horlogerie notamment), se juxtapose une seconde phase, inspirée par la composition palatiale de l'École polytechnique de Gottfried Semper à Zurich. Anoblie par cette référence, l'école professionnelle décline le thème sempérien sur un mode mineur, les dimensions et les matériaux en particulier avouant la prééminence de la "maison-mère", qui est d'ailleurs un modèle pour toutes sortes d'autres architectures en Suisse à l'époque. Alors que les fonctions spécifiques des écoles professionnelles se révèlent par des solutions architecturales typées (qui vont de l'usine au palais scolaire), les pistes se brouillent avec l'émergence du régionalisme qui, durant près d'un demi-siècle, enduit les écoles d'une esthétique patriotique qui change le message. De manière générale en Suisse, les édifices scolaires seront Heimatsil puis Landistil, quelle que soit leur destination. Ce n'est qu'à des détails parfois infimes - la sobriété appuyée des façades, le type d'œuvres rapportées à l'architecture, une salle de

87 Lenzbourg, Gewerbeschule, Zimmerli et Blattner, 1971; Rapperswil, Berufsschulzentrum, Kurt Federer, 1973-1976, etc. Cf. "Gewerbeschule Lenzburg", SBZ, vol.96/38, 1978, p.721-726; GAS, vol. 1, p. 104.

88 GAS, vol.3, p. 60; Pierre Frey (dir.), Heidi et Peter pour la vie : Wenger architectes, Lausanne, 2006, p. 115-116.

89 Notamment dans le monumental bâtiment des facultés des sciences humaines 2 de l'université de Lausanne en 1985-1987 (Bevilacqua, Dumas et Thibaud). 
gymnastique importante indiquant un établissement réservé aux seuls garçons, ce qui est alors rare - que l'école professionnelle peut s'affirmer. L'important pour les édiles est d'exprimer la fonction scolaire, quelle qu'elle soit. L'usure des solutions formelles produit une véritable rupture architecturale après les années 1950, au moment où l'appareil légal modifie en profondeur la nature même de l'enseignement. De plus en plus spécialisées, de plus en plus typées aussi, les écoles professionnelles reçoivent paradoxalement une architecture de plus en plus unifiée, d'influence corbuséenne d'une part - via le modèle majeur qu'est l'école des métiers de Berne, icône helvétique de l'architecture moderne des années 1930 - et miessienne d'autre part. La "sincérité" de l'un, la perfection de l'autre semblent pouvoir servir l'image des écoles professionnelles. En raison de la démocratisation des études universitaires, qui ont pour conséquence le déclassement symbolique des écoles des métiers, celles-ci doivent redorer leur blason. À nouveau, l'épuisement des formules - le thème corbuséen est utilisé des années 1930 à la fin des années 1970, Mies est à la mode des années 1950 à 1980 - appelle un renouveau formel qui se trouve confronté aux nouvelles tendances, contradictoires dans leurs intentions (sculpturalité abstraite du brutalisme, rigueur symétrique du post-modernisme), de la fin des Trente Glorieuses.

Au terme de ce panorama encore très grossier, les écoles formant l'essentiel de la population à une future carrière professionnelle apparaissent comme une véritable histoire simplifiée de l'évolution de l'architecture suisse des deux derniers siècles. Il reste à les mettre en relation avec les autres types d'architecture scolaire, dont l'histoire, si riche, attend toujours la synthèse qu'elle mérite.

Dave Lüthi

Université de Lausanne, section d'histoire de l'art, architecture \& patrimoine Dave.Luthi@unil.ch 